\title{
Calibration of antenna-radome and monument-multipath effect of GEONET-Part 1: Measurement of phase characteristics
}

\author{
Yuki Hatanaka, Masanori Sawada, Akiko Horita, and Masaaki Kusaka \\ Geographical Survey Institute, 1 Kitasato, Tsukuba, Ibaraki 305-0811, Japan
}

(Received May 15, 2000; Revised September 14, 2000; Accepted September 18, 2000)

\begin{abstract}
Severe, 10-cm level, elevation angle cutoff dependence of the height solution is detected in the analysis of sample baselines from GSI/GEONET (Geographical Survey Institute/GPS Earth Observation NETwork). It is inferred that station and monument specific differences in the radome and/or multipath environment are responsible for this effect, because it can be observed over short sample baselines for identical antennas mounted atop different monument types. Our test results show that both the radome and multipath from the metal plate at the top of the pillar affect the baseline solutions. A calibration experiment was carried out by observing short $(<10 \mathrm{~m})$ known baselines to obtain phase correction maps for the typical GEONET monuments. The antenna/monument phase centers of GEONET monument replicas with the same attachment and radomes but shortened in height, were determined relative to a tripod-mounted TRM29659.00 antenna. Phase maps were obtained with the BERNESE software for 6 typical combinations of 3 antenna types, 3 monument types, and 3 radome types commonly used in GEONET. We find monument/antenna specific phase differences up to $1 \mathrm{~cm}$. These phase differences can result in more than 10-cm station height biases when tropospheric delay parameters are estimated, which is consistent with the height errors observed for GEONET.
\end{abstract}

\section{Introduction}

The Global Positioning System (GPS) has been widely used as a powerful tool to observe various geophysical phenomena such as plate motion, crustal deformation, troposphere, ionosphere, etc. Although this is because GPS is sensitive to many types of signals, this also means, in turn, that GPS is sensitive to various sources of error. Therefore, the quality of the carrier phase observable, the primary GPS observable, is important to achieve the highest accuracy in applications of GPS in the Earth Sciences.

Recently, site-specific problems of GPS observation have received attention from researchers of precise application of GPS. Elósegui et al. (1995) and Jaldehag et al. (1996a) showed that the signal scattering from the top of the antenna pillar affects the phase observable and the estimate of vertical component of site position, and that putting electrical wave absorbing materials on the pillar is effective in reducing this problem. Carrier phase of GPS signals is also affected by radomes used for protecting GPS antennas, and thus affects the results of the analysis (Meertens et al., 1996; Johansson et al., 1998). This effect depends on the shape of the radomes for a given material. According to tests by Johansson et al. (1998), hemi-spherical radomes are less harmful than conical radomes. Jaldehag et al. (1996b) found that snow accumulated on GPS antennas significantly affect the estimates of site position.

GSI has been operating the nationwide permanent GPS network called GEONET (GPS Earth Observation NETwork;

Copy right (C) The Society of Geomagnetism and Earth, Planetary and Space Sciences (SGEPSS); The Seismological Society of Japan; The Volcanological Society of Japan; The Geodetic Society of Japan; The Japanese Society for Planetary Sciences.
Miyazaki et al., 1998). The number of stations is about 1000 as of January 2000. The GEONET data have been used for various applications in the area of geodesy, crustal deformation analysis, meteorology, science of ionosphere, etc. Since several types of antennas are mixed in the network, it was anticipated that there would be biases in the baseline solutions between stations with different antenna types due to the mis-modeling of antenna phase characteristics. Moreover, the phase maps for the antenna types used in GEONET were not available at the beginning of the operation. The phase maps for other antenna types that were provided by the International GPS Service (IGS) were substituted for GEONET sites; i.e. TRM14532.00 instead of TRM23903.00, ASH700228A instead of ASH700718A, and AOAD/M_T instead of LEIAT303. They are referred as "old phase maps" hereafter in this paper. To minimize these problems, the network was divided into three sub-networks so that the antenna types are uniform within each sub-network. Differences in monumentation such as radomes or antenna mount have, however, not received attention until recently.

In this paper, first we provide evidence of presence of un-modeled phase errors in GEONET due to site monumentation. Then we will try to obtain phase characteristics of GEONET monuments from a phase calibration experiment.

\section{Examples of Phase Modeling Errors in GEONET}

To assess the stability and consistency of the baseline solutions, a $286 \mathrm{~m}$ baseline was analyzed with various elevation cutoff angles from 10 degrees to 25 degrees and with various analysis strategies. Both stations of this baseline are 

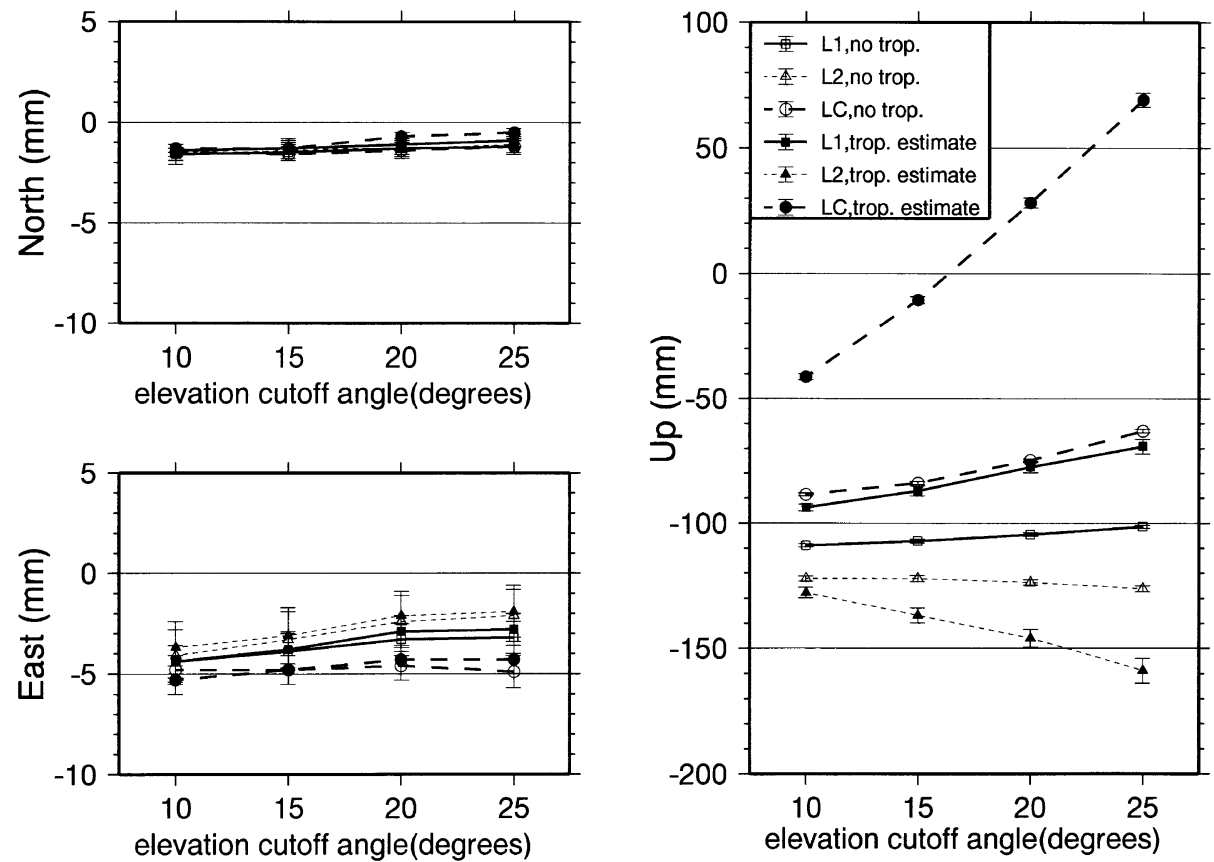

Fig. 1. Results of test analysis of a $286 \mathrm{~m}$ baseline of GEONET (between the sites 92110 and 960627 ) at the GSI's campus with various analysis strategies. L1, L2, and LC phase observables are used, respectively, with and without estimating tropospheric delay. The elevation cutoff angle is changed from 10 degrees to 25 degrees. The deviation from the routine solution of GEONET are plotted.

GEONET sites, are located in the GSI's campus in Tsukuba, and are equipped with Trimble antennas of the same type (TRM23903.00). Since the baseline length is very short, the phase delay due to troposphere and ionosphere can be regarded approximately as equal for both sites and are canceled out when a double-difference observable is formed (e.g. Leick, 1995). The data are analyzed with various strategies: each of L1, L2, and LC (ionosphere-free linear combination) observable is analyzed with and without estimating troposphere zenith delay. (For a description of the GPS observable, see e.g. Rothacher and Mervart, 1996; Leick, 1995.)

The results (Fig. 1) show that the vertical component of solutions is not constant but depends on the elevation cutoff angle and the analysis strategy by $20-\mathrm{cm}$ level. The change in the solutions caused by changing the "elevation mask" is significantly large when LC is used and tropospheric delay is estimated. These phenomena are indications of existence of un-modeled phase errors which depend on the elevation angle of the satellite (e.g. Elósegui et al., 1995). This is analogous to the case of modeling error of antenna phase characteristics (e.g. Meertens et al., 1996; Mader, 1999). Because of strong correlation among the parameters, the vertical coordinates become more unstable and are more affected by un-modeled errors when the troposphere zenith delay parameters are estimated, instead of the benefit of eliminating troposphere noise (e.g. Beutler, 1998). The large bias in the vertical coordinates between different observables implies that the relative position between L1 and L2 phase centers is not the same for the two sites.

Since the antennas used at both sites are the same type in this case, the antenna phase characteristics are not considered to be the cause. Therefore, the cause of the un-modeled phase errors is something else but has an effect that is similar to the antenna phase problems, which is not canceled out by differencing technique. Since the types of pillars and radomes are only the differences between the 2 stations of the baseline, the difference in the monumentation is plausible to be the cause.

Another evidence of deterioration of phase is seen in the phase residuals after least-squares estimation in the network analysis of GEONET. Figures 2(a) and 2(b) show doubledifference phase residuals of 6 baselines to 1 common site and satellite pair taken from the routine analysis. Again, the antenna type, ASH700718A, is the same for the all sites, however, the baselines in Fig. 2(b) are between old monuments built in 1994 and newer ones built after 1995 while the all monuments of the baselines in Fig. 2(a) are built in 1994. The residuals in Fig. 2(b) clearly show systematic wavy patterns whose amplitude is about $5 \mathrm{~cm}$, while Fig. 2(a) does not show particular feature except for high noise level at the ends of the data arc where the elevation angle of a satellite is low. These systematic patterns are direct evidences of un-modeled phase error. Since these patterns are seen only for the baselines between the old monuments and the newer ones, it is inferred that the phase errors depend on the monument types and that the errors are not canceled out when the monument types are different. There are several differences between the 2 monument types, such as material of the radome, structure of the pillar top, the way of antenna mounting, etc. The difference in the antenna mount results in the different antenna height from the wide metal plate beneath the antenna. Since the effect of the signal scattering from the near-field reflector (Elósegui et al., 1995) is sensitive to the distance from the reflector, this difference can produce different multipath 
(a)
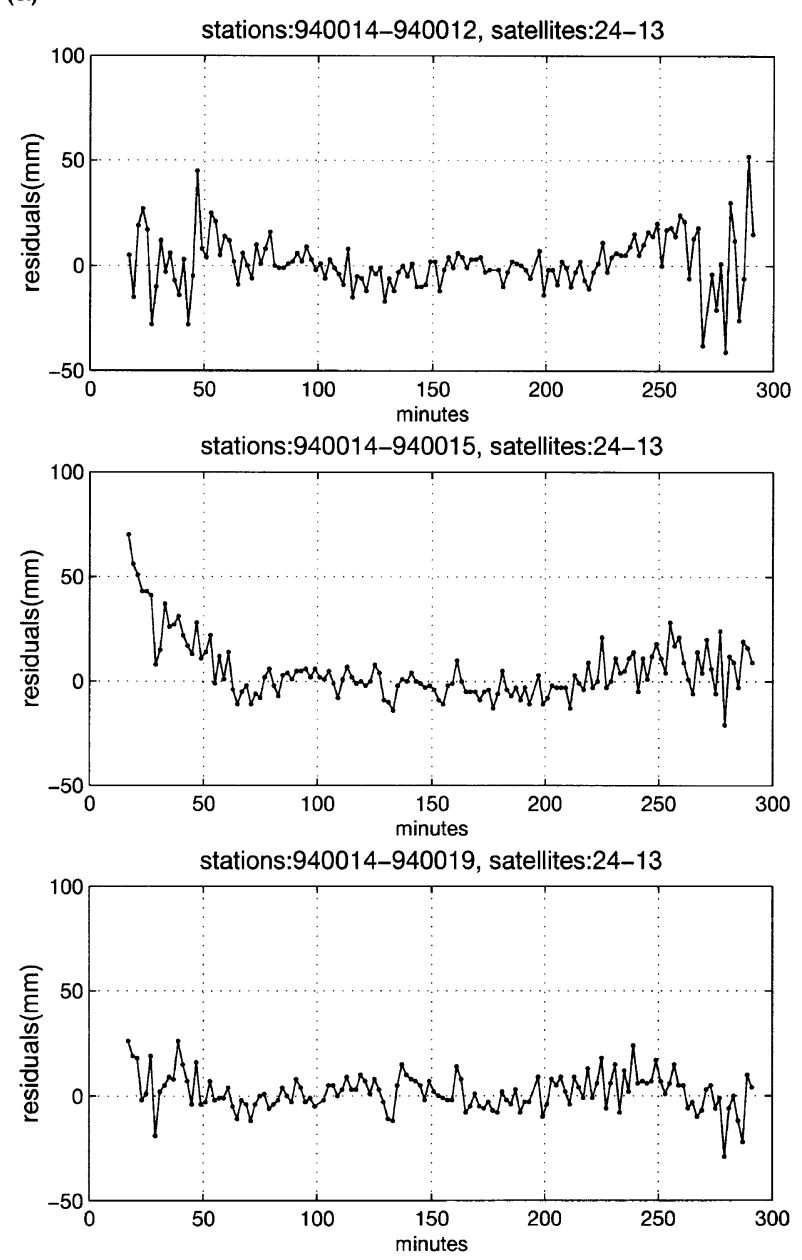

(b)
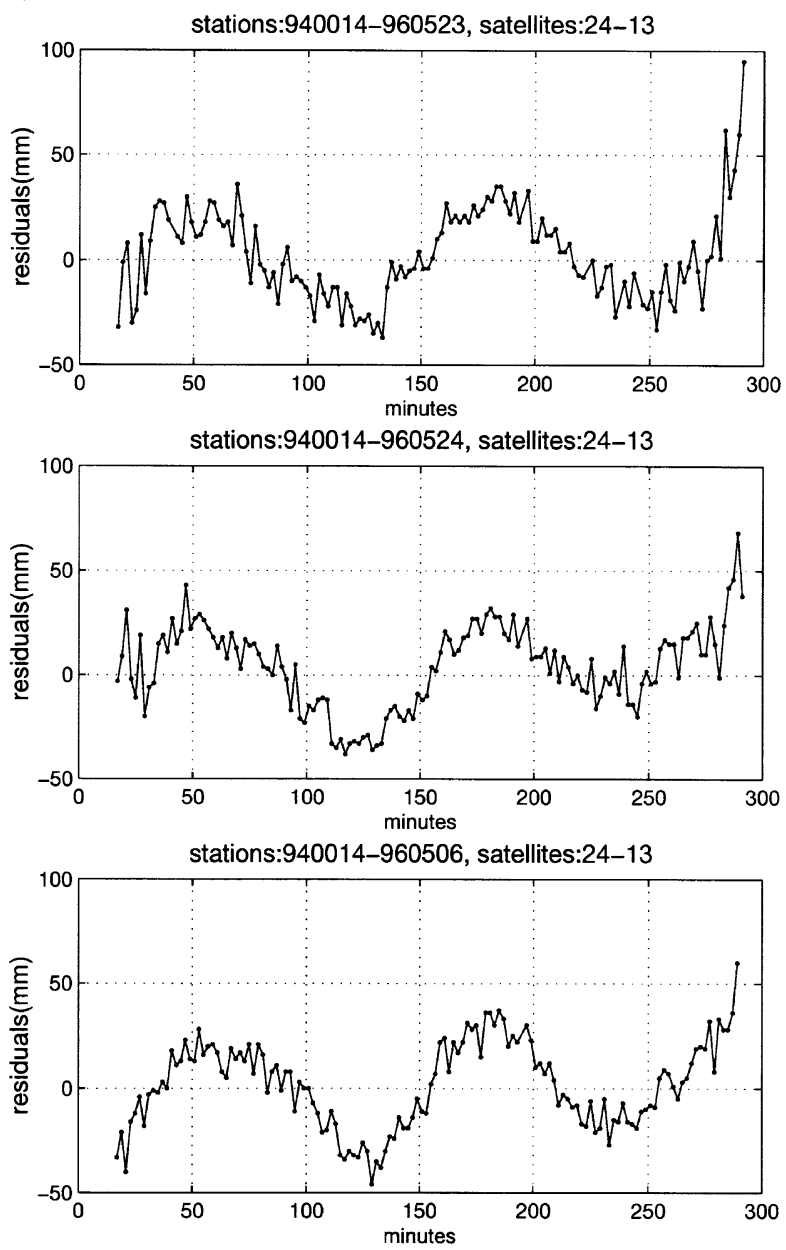

Fig. 2. (a) Samples of double difference phase residuals taken from the routine analysis of GEONET for the baselines with the same monuments and (b) those for the baselines between old and new monuments. The origin of horizontal axis is 9:00:00 UT, Apr. 29, 1998.

environment.

These lines of evidence clearly demonstrate that there are differences in the phase characteristics between different monument types even with the same antenna types and that such differences affect the baseline solutions at the $10-\mathrm{cm}$ level or larger. Because the systematic pattern shown in the residuals in Fig. 2(b) is similar between different baselines, it is expected that the phase errors are specific for monument types, i.e. the phase errors depend on the monument types but is similar if the monument types are the same. If this is the case, it is possible to calibrate the phase characteristics by applying phase correction specific for each monument type.

\section{Classification of GEONET Stations}

Because of changes of site specifications during the construction history of the GEONET network, there are several different types of stations within the network; 3 antenna types, 3 pillar types, and several radome types. The phase characteristics can be different by any different combination of these conditions. Most of the stations are, however, classified into 6 typical combinations as listed in Table 1, though there are some exceptions.
Table 1. List of specification of typical GEONET monuments.

\begin{tabular}{cccl}
\hline Type No. & Antenna type & Pillar type & Radome type \\
\hline$\# 1$ & TRM23903.00 & Model 93 & conical \\
$\# 2$ & TRM23903.00 & Model 93 & None \\
$\# 3$ & TRM23903.00 & Model 95 & hemisphere 2 \\
$\# 4$ & ASH700718A & Model 94 & hemisphere 1 \\
$\# 5$ & ASH700718A & Model 95 & hemisphere 2 \\
$\# 6$ & LEIAT303 & Model 95 & hemisphere 2 \\
\hline
\end{tabular}

\section{Measurement of Phase Characteristics}

An observation campaign was carried out to measure the phase characteristics of each type of GEONET station. Three special monuments of $1.5-\mathrm{m}$ tall were made. Each of them has the same top structure as respective monument type of actual GEONET stations. Radomes can be put on or off according to the monument type to examine. By setting these monuments with the appropriate antennas, we reproduced the observation environment of the actual GEONET monuments to first order.

Since double-difference phase observables are used for 
the analysis to eliminate satellite and receiver clocks, the phase characteristics are solved not in absolute sense, but the difference of the phase characteristics between two sites are resolved. Therefore, a reference antenna is necessary for the observation. A Trimble choke ring antenna (TRM29659.00) was used as a reference antenna, and was put on a tripod. The phase characteristics of each monument type will be measured relative to this antenna. Since all the sites including the reference antenna are set within an area of $10 \mathrm{~m}$, the troposphere and ionosphere noises are canceled out by the double difference operation. The observation was carried out at least for 24 hours for each setting of the station types.

The BERNESE software (Rothacher and Mervart, 1996) was used for the analysis. At first, the mean phase centers were solved with a minimum elevation angle of 15 degrees that is often adopted in surveys by GPS. Then, phase center variations (PCV) around the mean phase centers were solved. The PCVs were modeled with spherical harmonics expansion in azimuth and elevation angle (Rothacher et al., 1995a, b). Data from below 10 degrees elevation were not used for the analysis of PCV because of higher multipath noise level and fewer data due to limitation of satellite visibility. Throughout the analysis, the baseline components from the reference antenna to each testing monuments were fixed to the values that were measured in previous by leveling, EDM, and GPS with uniform antenna type (TRM23903.00) and with putting electrical wave absorbing materials on the pillars. The phase characteristics of the reference antenna were fixed to the nominal value currently adopted by the International GPS Service (IGS), i.e. the height of phase center of $110 \mathrm{~mm}$ for L1 phase and $128 \mathrm{~mm}$ for L2 phase above the antenna reference point (bottom of preamplifier), and no phase center variation (the official antenna phase table of IGS is available from IGS Central Bureau; ftp://igscb.jpl.nasa.gov/igscb/ station/general/igs_01.pcv). The obtained phase maps should contain the total effect of signal scattering from the pillars, phase delay due to radomes, and the antenna phase characteristics.

It is important to define the antenna reference point clearly since the mean phase centers are measured from the antenna reference points. In this study, they are at the bottom of preamplifier for the Trimble and Ashtech stations which is the same definition as in the IGS. For the Leica stations, a 45$\mathrm{mm}$ spacer is put under the antenna because of the structure of the antenna/monument. The reference point for the Leica stations is defined at $45 \mathrm{~mm}$ below the bottom of preamplifier, i.e. bottom of the spacer. This definition is different from that by the IGS, and is taken to avoid the change of definition of marker position and antenna height in case of replacement of antennas with removing the spacers.

\section{Phase Characteristics Estimated from the Cal- ibration Campaign}

The mean phase center offsets obtained from the experiment are listed in Table 2. The offsets are measured from the antenna reference point defined in the previous section. The values for Type \#1 and Type \#2 monuments are set to be the same since their differences are insignificant (less than $1 \mathrm{~mm})$. While the differences of the horizontal offsets are within $1 \mathrm{~mm}$ for the same antenna types, the vertical offsets
Table 2. Mean phase center for elevation mask of 15 degrees

\begin{tabular}{crrrrrrrr}
\hline & \multicolumn{3}{c}{ L1 phase $(\mathrm{mm})$} & & \multicolumn{3}{c}{ L2 phase $(\mathrm{mm})$} \\
\cline { 2 - 4 } \cline { 7 - 8 } Type No. & North & East & Up & & North & East & Up \\
\hline$\# 1$ & 1.4 & -1.7 & 75.6 & & -1.1 & 1.4 & 74.6 \\
$\# 2$ & 1.4 & -1.7 & 75.6 & & -1.1 & 1.4 & 74.6 \\
$\# 3$ & 1.1 & -2.1 & 79.4 & & -0.7 & 1.1 & 71.7 \\
$\# 4$ & -3.1 & -0.7 & 78.7 & & -1.8 & -0.7 & 68.5 \\
$\# 5$ & -3.9 & -0.4 & 88.5 & & -0.8 & -0.4 & 64.2 \\
$\# 6$ & 0.7 & 2.1 & 74.2 & & 1.1 & 0.4 & 88.6 \\
\hline
\end{tabular}

are clearly different even if the antenna types are the same. For the Trimble sites, the position of the mean phase centers of Type \#3 is higher by $3.8 \mathrm{~mm}$ for L1 phase and lower by $2.9 \mathrm{~mm}$ for L2 phase than those of Type \#1 and Type \#2. The corresponding difference of LC phase center is $14.2 \mathrm{~mm}$ that is calculated by the following expression (e.g. Beutler et al., 1989; Rothacher and Mervart, 1996):

$$
\begin{aligned}
d(\mathrm{LC})= & \frac{f_{1}^{2}}{f_{1}^{2}-f_{2}^{2}} d(\mathrm{~L} 1)-\frac{f_{2}^{2}}{f_{1}^{2}-f_{2}^{2}} d(\mathrm{~L} 2) \\
& \approx 2.55 d(\mathrm{~L} 1)-1.55 d(\mathrm{~L} 2)
\end{aligned}
$$

where $f_{1}$ and $f_{2}$ are the frequency of the L1 and L 2 carriers, respectively. For the Ashtech sites, the position of the mean phase centers of Type \#5 is higher by $9.8 \mathrm{~mm}$ for L1 phase, lower by $4.3 \mathrm{~mm}$ for L2 phase, and higher by $31.7 \mathrm{~mm}$ for LC phase than those of Type \#4.

The phase shift due to the phase characteristics of each monument type is mapped around the corresponding phase center in Table 2. 2 types of phase mapping models are used for the analysis; one as a function of elevation angle and azimuth, the other as a function of elevation angle only.

Figure 3 shows the phase maps as functions of elevation angle and azimuth. Some of the figures show large values at the north where the solutions are unstable due to lack of data at the hole in the satellites coverage. The azimuthal variation is small for the Trimble types and the Leica types. Aside from the "north hole", the variation is typically within $6 \mathrm{~mm}$ in azimuth above the elevation angle of 15 degrees, therefore, an elevation dependent phase model is adequate for these station types. Systematic azimuthal patterns are seen in the phase maps for the Ashtech monuments. The azimuthal variation exceeds $2 \mathrm{~cm}$. It is inferred that these patterns come from the phase characteristics of the antenna since the phase characteristics of this antenna type measured in an anechoic chamber (Imakiire et al., 1997) shows similar pattern. It is clear from this figure that the azimuthal variation should be taken into account for the perfect calibration of the phase characteristics of the Ashtech stations. However, we decided to use elevation-dependent phase maps instead of these phase maps for two reasons: (1) The phase maps with azimuthal variation have extreme values at the "north hole" of satellite coverage, showing that the solutions are unstable where the data is sparse. Since the satellite coverage varies with the location (latitude) of the site, the extreme values can cause a problem if satellites cross this area in the sky. This could happen at locations of different latitude from the 


\section{(a)Trimble Antennas with GEONET Monuments}
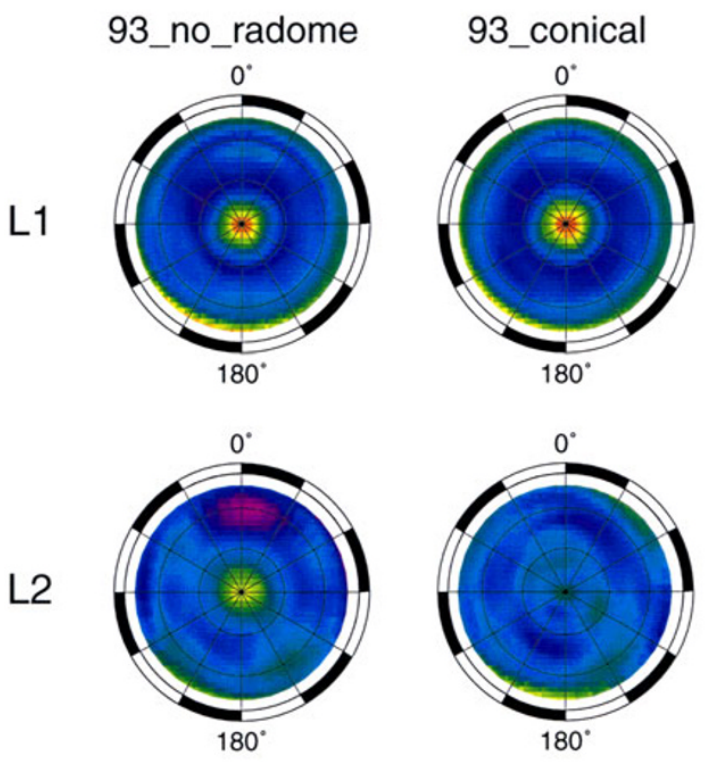

\section{5_spherical}

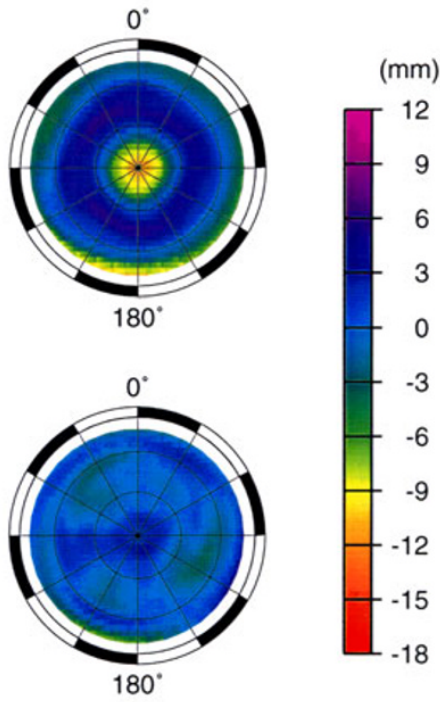

(b)Ashtech Antennas with GEONET Monuments

(c)Leica Antennas with GEONET Monuments
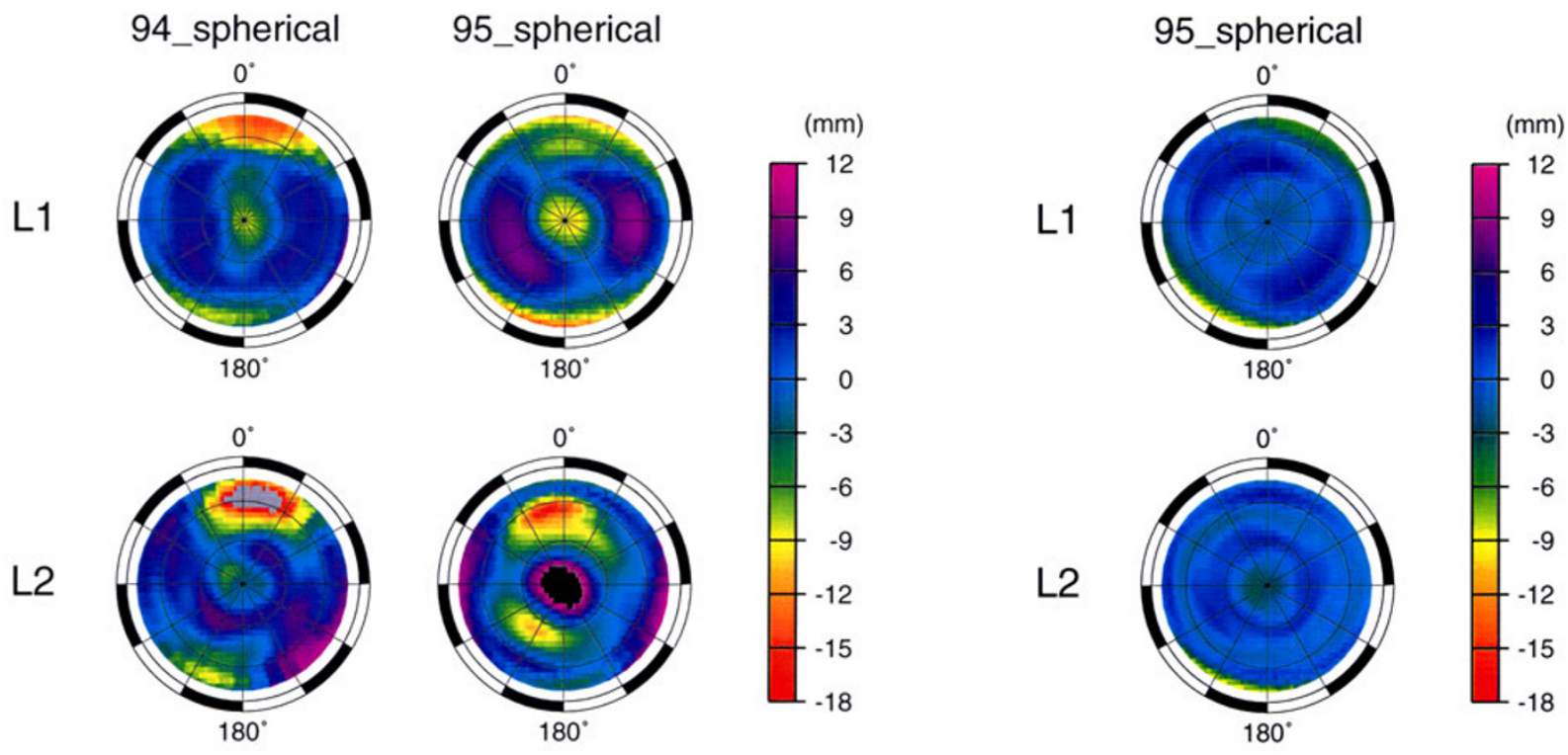

Fig. 3. The maps of phase shift as functions of elevation angle and azimuth of the satellites for the monument types (a) with Trimble antennas (Type \#1-3), (b) for that with the Ashtech antennas (Type \#4, \#5), and (c) for that with the Leica antennas (Type \#6). The gray area in the left bottom panel and the black area in the right bottom panel of (b) indicate the value less than $-18 \mathrm{~mm}$ and that larger than $12 \mathrm{~mm}$, respectively.

Tsukuba where the calibration experiment was carried out. (2) The antenna may not be precisely oriented to the right direction at every station. If the antenna orientation is not correct, the azimuthal variation of the phase correction is not effective, or in worst case, it could bring errors into the solution. The effect of neglecting the azimuthal pattern would be less significant for the repeatability of daily solutions since effect of the azimuthal variation would be averaged. However, it could be a problem for kinematic analysis in which instantaneous phase error is not effectively averaged out. The azimuthal phase pattern may be applied when the antenna orientation is checked in future, or when in situ calibration is carried out site by site. These are not done in this study.

Figure 4 shows the PCV curves for each station type as a function of elevation angle. The PCV curves for LC (ionosphere-free linear combination) phase are composed from those for L1 and L2 phase by the Eq. (1). It is evident from these plots that the phase curves are different by the monument types for the Trimble- and the Ashtech-monuments. The difference is larger for L2 phase than L1 phase and is magnified when the LC phases (ionosphere-free linear combination) are formed. The monument type \#1 has the radome of conical shape while the type \#2 has no radome. The corresponding curves shows difference at high elevation angle 
(a)Trimble monuments
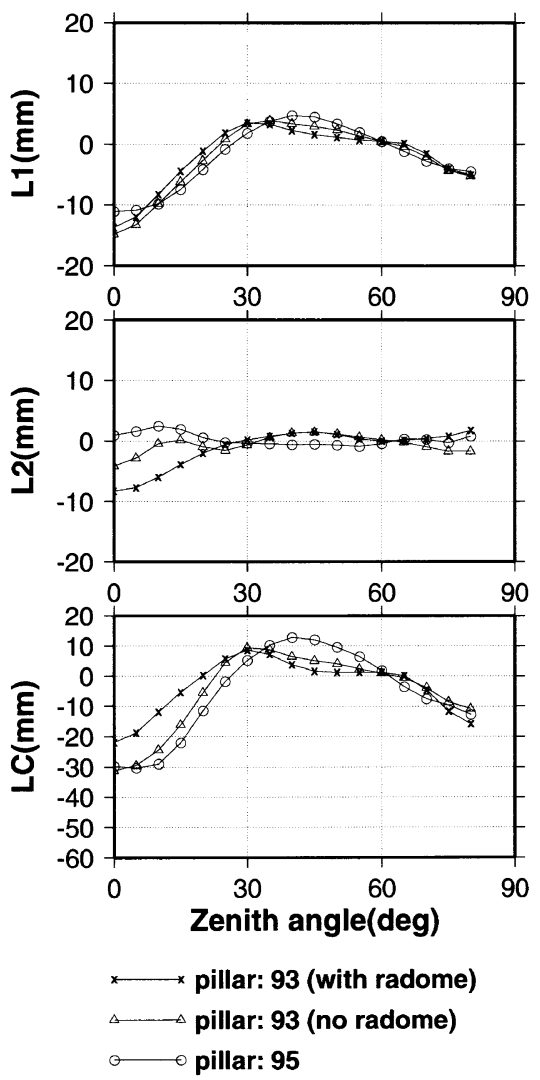

(b)Ashtech monuments
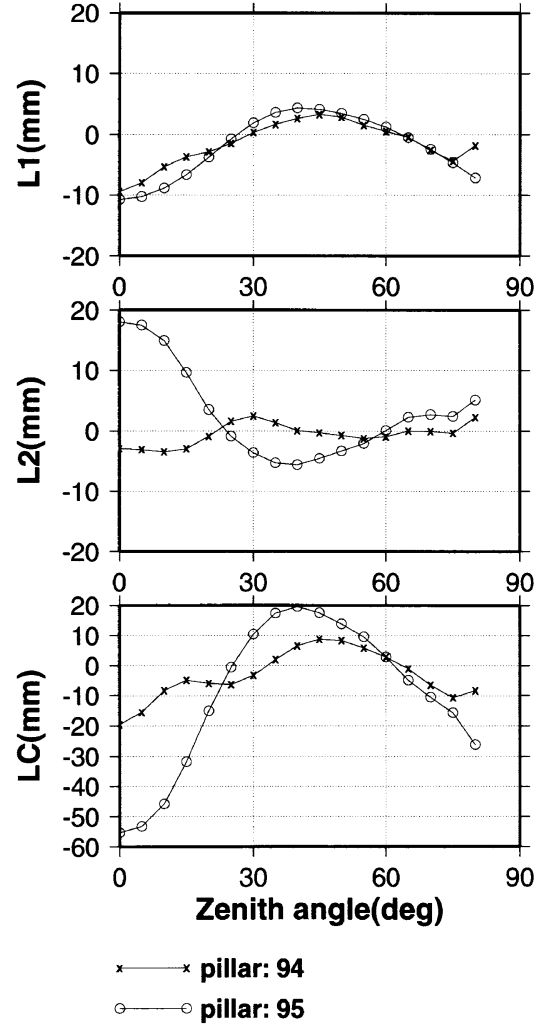

(c)Leica monuments
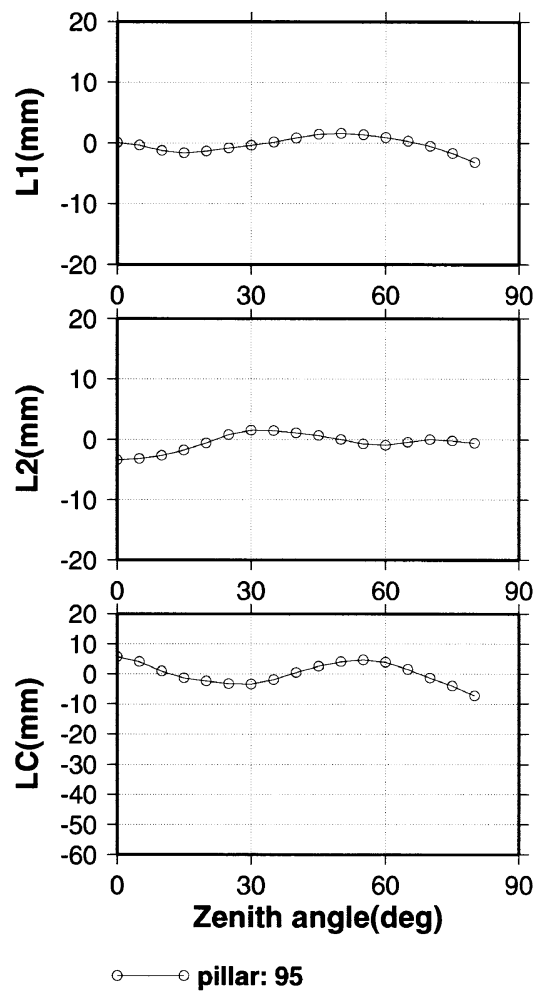

Fig. 4. The phase plots as functions of elevation angle of the satellites for the monument types (a) with Trimble antennas (Type \#1-3), (b) for that with the Ashtech monuments (Type \#4, \#5), and (c) for that with the Leica antennas (Type \#6).

which is considered to be caused mainly by the radome.

In spite of the fact that both monument types with Ashtech antennas are equipped with the hemi-spherical radomes, the phase maps of these monument types are significantly different, especially, for L2 phase (and consequently LC phase). Although the materials of the radomes are also different, this effect is inferred to be smaller than in the case of conical radome since the hemi-spherical radome is more symmetric. Therefore, the difference in the top structure of the pillar, i.e. the characteristics of the signal scattering from the top of the pillar, is inferred to be the main cause of the difference in the phase maps.

We conclude that the differences in both the radomes and the pillars are causing the un-modeled phase errors.

\section{The Effect of the Correction by the New Phase Maps}

To evaluate the effects of the new phase maps, the same baseline as in the Fig. 1 is analyzed in the same way but by applying the new phase maps (Fig. 5) for the correction. The height component of the LC solution with tropospheric delay estimation becomes consistent within a few centimeter level. The other solutions are consistent in 1-cm level. Assuming that the new phase maps provide the correct solution, the systematic error in the LC in the old solutions is about $7 \mathrm{~cm}$ for elevation cutoff angle of 10 degrees and 18 $\mathrm{cm}$ for 25 degrees when the troposphere is estimated. This huge inconsistency is almost eliminated by taking into account the small difference in LC phase model less than $2 \mathrm{~cm}$. The difference in the L1 and L2 phase model is only several millimeter level. These results demonstrate how large systematic errors in vertical coordinate are caused by small errors in the phase models.

Figure 6 is the similar results to Fig. 1, but the elevation dependent data weighting (sigma is proportional to cosine of zenith angle) is applied as in the case of current routine analysis of GEONET system. The discrepancies among solutions are larger than that in the case of Fig. 1 by a few centimeters at lower elevation cutoff angle. This figure demonstrates again that the height coordinates are sensitive to the change in the analysis strategy. In the routine analysis of GEONET, LC phase is used, the troposphere is estimated, and the elevation cutoff mask is 15 degrees. In this case, a height bias of about $12 \mathrm{~cm}$ is expected to be in the baseline solutions.

\section{Discussion}

The LC solutions with troposphere estimation using old phase maps (Fig. 6) show the largest deviation from the others and significantly vary with the elevation cutoff angle by up to $10-\mathrm{cm}$ level. These plots clearly demonstrate that the GPS solutions can be significantly degraded by the phase modeling error due to the difference of monuments even if the antenna types are the same. These results imply not only that the phase modeling error brings large systematic errors 

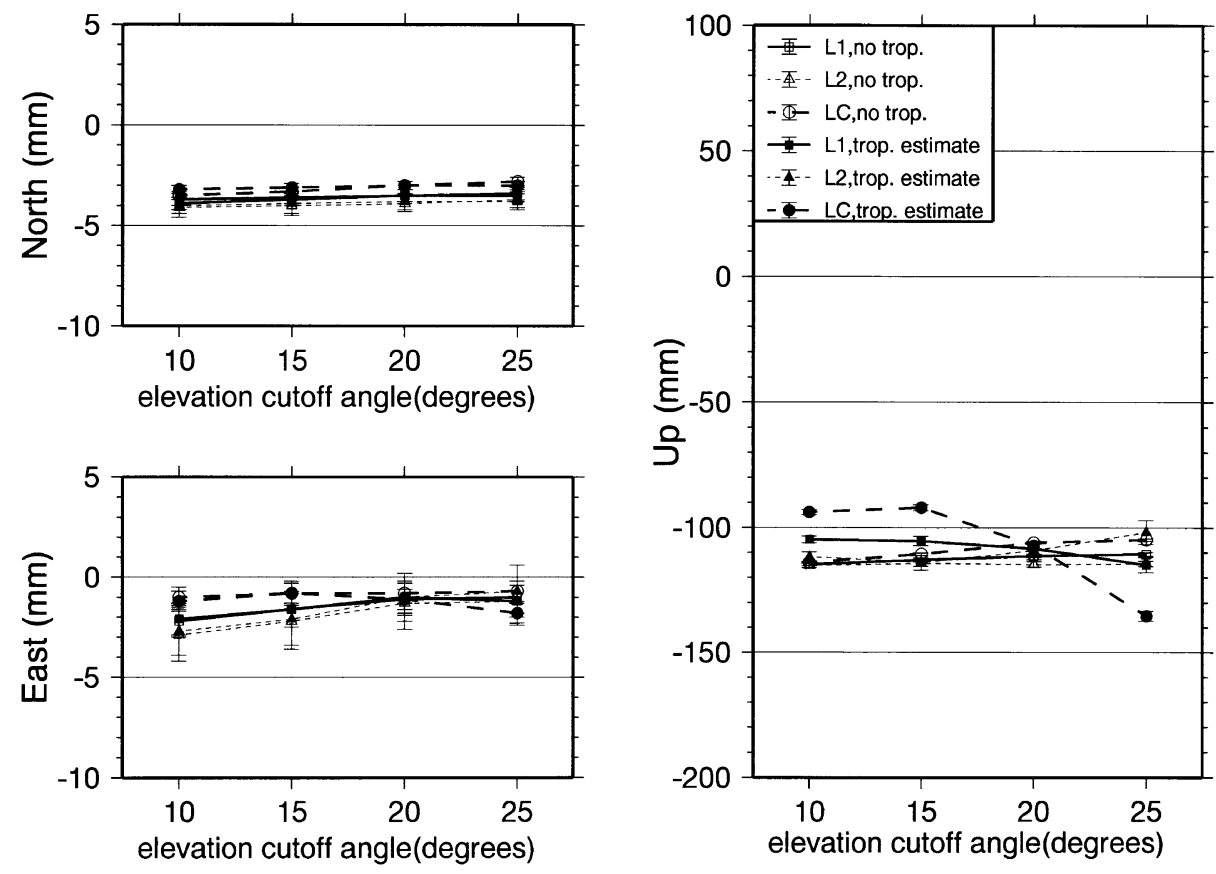

Fig. 5. The results of the test analysis of the same data as that in Fig. 1 but with the new phase maps shown in Fig. 4.
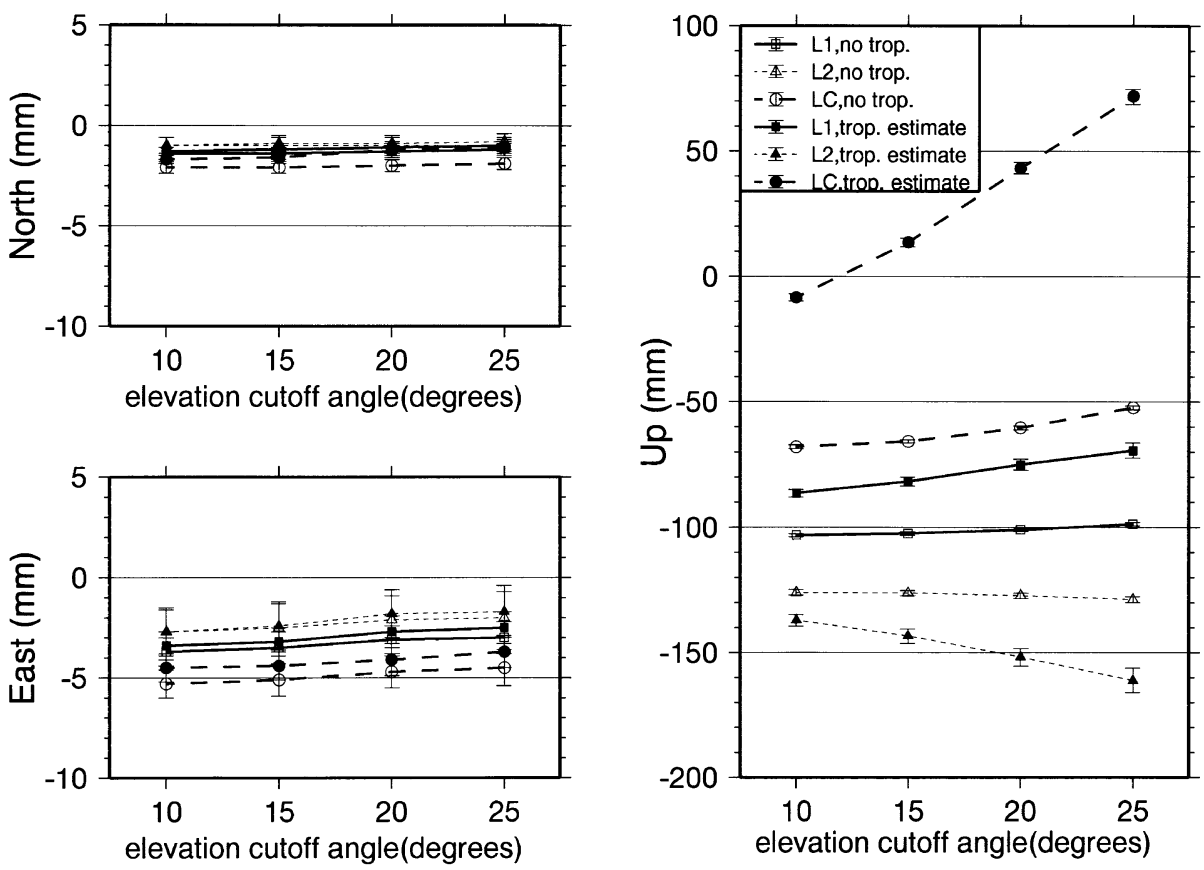

Fig. 6. The results of the test analysis of the same data as that in Fig. 1 with applying elevation-dependent data weighting.

into the solutions, but also that the size of the systematic errors depends on the analysis strategy. Since the consistency of GPS solutions is spoiled by this fact, baseline solutions obtained by different strategy should not be compared directly. This has big implications for the use of GEONET as geodetic control stations. In fact, comparison of Figs. 1 and 5 proves that the LC solution with estimating troposphere, which is the case of GEONET routine analysis, has a height bias of more than $10 \mathrm{~cm}$ unless the monument-dependent phase maps are applied, while the bias of the L1 solutions without estimating troposphere, which is the usual method for the analysis of short baselines within a few kilometers, is much smaller. If the geodetic reference frame is realized without correcting the LC solutions for the monument-dependent phase characteristics, users of single frequency receivers will find a discrepancy of $10 \mathrm{~cm}$ in the vertical component by comparing 

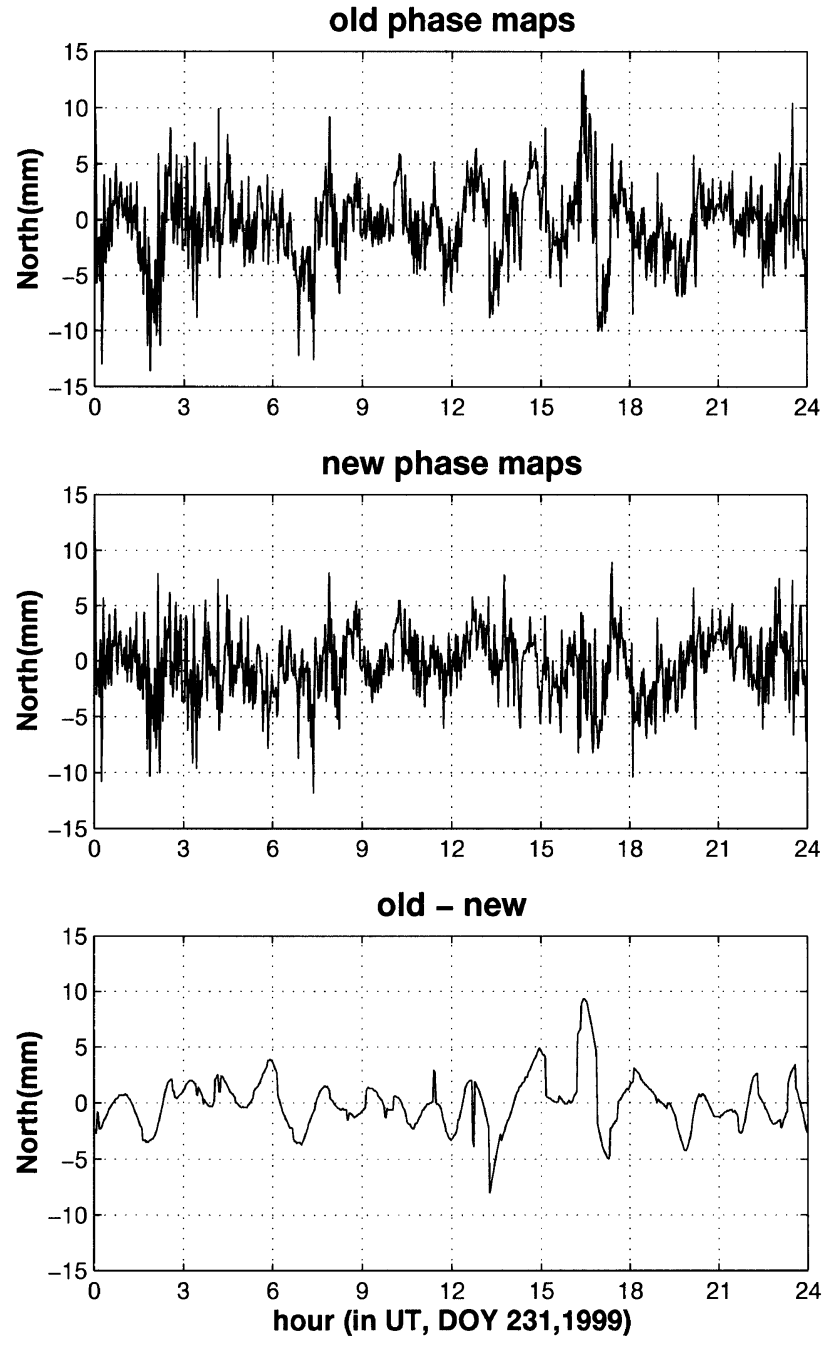

Fig. 7. Epoch-to-epoch solutions (every 2 minutes) of a $286 \mathrm{~m}$ baseline at GSI (the same baseline as that in Fig. 1) analyzed with old phase maps (top) and new phase maps (middle), respectively, and the difference of them (bottom). Only the north-south component is shown as an example.

the solutions of baselines from a rover site to two GEONET sites with different monument types.

Modeling error in phase characteristics affects more severely GPS applications which need high temporal resolution such as GPS kinematic analysis. Figure 7 shows a timeseries of the epoch-to-epoch solutions of a short baseline (of the same baseline as that in Fig. 1) analyzed with kinematic method by using L1 phase with the old phase maps (top) and new phase maps (middle), respectively. Since most of the troposphere noise is canceled because the baseline length is very short $(286 \mathrm{~m})$, the epoch-to-epoch solutions should be constant in ideal conditions. It is clear from Fig. 7 that the noise in the time series is not random, and that the low frequency noise in the solutions using the old phase maps is reduced in the solutions using the new phase maps. The values of standard deviation (S.D.) of the epoch-to-epoch coordinates (Table 3 ) prove the reduction of S.D. values by $0.40-0.56 \mathrm{~mm}$. The difference between two coordinate sets (bottom of Fig. 7) shows a staggering motion with the timescale of several tens of minutes. Note that no smoothing
Table 3. Standard deviation of epoch-to-epoch coordinates (L1 solutions) and their difference in millimeters.

\begin{tabular}{lccc}
\hline & NS & EW & UD \\
\hline old phase maps & 3.72 & 3.21 & 7.38 \\
new phase maps & 3.17 & 2.81 & 6.93 \\
\hline S.D. reduction & 0.56 & 0.40 & 0.46 \\
S.D. of difference & 2.29 & 1.55 & 4.73 \\
\hline
\end{tabular}

is applied for this plot. The difference in the old and new PCV models affects only the low frequency component of the coordinate time series. The high frequency component of noise in the kinematic solutions may not be explained by these PCV models. The amount of the difference is several millimeters for the north-south component. The S.D. values of this difference, listed in the bottom line of Table 3, reach several millimeters. The maximum values are two to three times larger than the S.D. values (compare bottom of Fig. 7 and bottom line of the Table 3 ). This difference can be regarded as an artificial movement due to the modeling error of the phase characteristics if we tentatively suppose that the solution analyzed by the new phase maps is noise-free. This artificial noise (or a fake signal) is a problem if we are trying to detect rapid crustal movements of a few centimeter level with about one hour period, especially, in real-time, which could happen in volcano monitoring. For such applications, calibration of phase characteristics is mandatory if we mix different antennas or monuments.

The low frequency noise that is still remaining in the epoch-to-epoch solutions by the new phase maps (middle of Fig. 7) may indicate a limitation of the new phase maps. This may be caused by errors that still remain in the new phase maps or the difference between the site environment of the calibration experiment and that of the actual GEONET sites. In reality, the structure of the monument (such as the thickness of the radome and the antenna height from the metal plate beneath it) may not be accurately the same. Moreover, the baseline solutions may be affected not only by phase characteristics of antenna/monument, but also by environmental factors such as multipath. It is difficult to guarantee the perfect homogeneity of phase characteristics among the all GEONET sites even with the same monument types. Such site-specific noise is hard to eliminate perfectly by a calibration experiment carried out in a particular environment. In spite of those limitations, it is evident from the statistics in Table 3 that the new phase maps are much better models than the old ones.

\section{Conclusions}

The results of elevation cutoff test of a sample baseline of GEONET and the phase residuals in the GEONET routine solutions shows that un-modeled phase error of several centimeter level exists in the GEONET system even if the antenna types are the same for the both ends of the baselines. From the correlation of phase residuals for the same combination of the monument types, it is inferred that the error originates from a structure of the monument such as signal scattering from the top of the pillar and/or phase delay due 
to radome, and the possibility of phase calibration according to the monument types is expected to be effective.

Phase maps for typical monument types are obtained from the calibration experiment carried out using model pillars and the TRM29659.00 antenna as a reference. By applying the new phase maps, the situation is dramatically improved for the sample baseline. The baseline solutions become consistent within a few centimeters level and the low frequency noise in epoch-to-epoch solutions are reduced. The phase maps obtained in this study are useful for realization of geodetic reference frame and for geophysical application of GEONET such as GPS meteorology or geoid modeling through GPS leveling.

Although the new phase maps are obtained by using the models of real pillars, the results of the test encourage us to apply them to the actual monuments of GEONET. However, since only one sample baseline was used for the test in this paper, a question remains whether the new phase maps are also valid for other baselines, or in other words, whether the phase characteristics of GEONET stations are uniform for each monument type. The applicability of the new phase map to the whole GEONET network is extensively examined in the Part 2 of this paper (Hatanaka et al., 2001).

Acknowledgments. This study was partly supported by Science and Technology Agency of Japanese Government under the Special Coordination Funds for the Science on GPS meteorology project.

\section{References}

Beutler, G., The role of GPS in space geodesy, in GPS for Geodesy, Chapter 16, 2nd edition, edited by P. J. G. Tenuissen and A. Kleusberg, pp. 626650, Springer, 1998.

Beutler, G., W. Gurtner, M. Rothacher, U. Wild, and E. Frei, Relative static positioning with the Global Positioning System: Basic technical considerations, in Global Positioning System: An Overview, edited by Y. Bock and N. Leppard, International Association of Geodesy Symposium No. 102, ISBN 3-540-97266-8, pp. 1-23, 1989.

Elósegui, P., J. L. Davis, R. T. K. Jaldehag, J. M. Johansson, A. E. Niell, and I. I. Shapiro, Geodesy using the Global Positioning System: The effect of signal scattering on estimates of site position, J. Geophys. Res., 100, 9921-9934, 1995.
Hatanaka, Y., M. Sawada, A. Horita, M. Kusaka, J. M. Johnson, and C. Rocken, Calibration of antenna-radome and monument-multipath effect of GEONET-Part 2: Evaluation of the phase map by GEONET data, Earth Planets Space, 53, this issue, 23-30, 2001.

Imakiire, T., Y. Iimura, S. Saga, H. Morishita, A. Tsuchiya, F. Kudo, S. Ishikawa, and Y. Iwana, Results of phase characteristics measurements of GPS receiver antennas (2), J. Geod. Soc. Japan, 43, 79-90, 1997.

Jaldehag, R. T. K., J. M. Johansson, B. O. Rönnäng, P. Elósegui, J. L. Davis, I. I. Shapiro, and A. E. Niell, Geodesy using the Swedish permanent GPS network: Effect of signal scattering on estimates of relative site positions, J. Geophys. Res., 101, 17841-17860, 1996a.

Jaldehag, R. T. K., J. M. Johansson, J. L. Davis, and P. Elósegui, Geodesy using the Swedish permanent GPS network: Effects of snow accumulation on estimation of site positions, Geophys. Res. Lett., 23, 1601-1604, $1996 b$.

Johansson, J. M., T. R. Carlsson, P. O. J. Jarlemark, L. P. Gradinarsky, and G. Elgered, The atmospheric influence on the results from the Swedish GPS network, Physics and Chemistry of the Earth, 23, 107-112, 1998.

Leick, A., GPS Satellite Surveying, 2nd edition, 560 pp., John Wiley \& Sons, Inc., New York, 1995.

Mader, G., GPS Antenna Calibration at National Geodetic Survey, GPS Solutions, 3, 50-58, 1999.

Meertens, C., C. Alber, J. Brown, C. Rocken, B. Stephens, R. Ware, M. Exner, and P. Kolesnikoff, Field and Anechoic Chamber test, Proceedings of IGS analysis Center Workshop, Silver Springs, MD, U.S.A., June 1996, International GPS Service, pp. 107-118, 1996.

Miyazaki, S., Y. Hatanaka, T. Sagiya, and T. Tada, The nationwide GPS array as an Earth observation system, Bull. Geogr. Surv. Inst., 44, 11-22, 1998.

Rothacher, M. and L. Mervart (eds.), Documentation of the Bernese GPS Software Version 4.0, 418 pp., Astronomical Institute, University of Berne, 1996.

Rothacher, M., W. Gurtner, S. Schaer, R. Weber, W. Schluter, and H. O. Hase, Azimuth- and elevation-dependent phase center corrections for geodetic GPS antennas estimated from GPS calibration campaigns, in GPS trends in Precise Terrestrial, Airborne, and Spacebourne Applications, edited by G. Beutler, G. H. Hein, W. G. Melbourne, and G. Seeber, International Association of Geodesy Symposium No. 115, ISBN 3-54060872-9, pp. 333-338, 1995a.

Rothacher, M., S. Schaer, L. Mervart, and G. Beutler, Determination of antenna phase center variations using GPS data, in IGS Workshop Proceedings on Special Topics and New Directions, edited by G. Gendt and G. Dick, pp. 77-92, GeoForschungsZentrum, Potsdam, Germany, May 15-18 1995, 1995b.

Y. Hatanaka (e-mail: hata@gsi.go.jp), M. Sawada, A. Horita, and M. Kusaka 